\title{
Total antioxidant capacity of 10 commercially available tomato juices before and after in vitro digestion
}

\author{
P. C. Wootton-Beard and L. Ryan \\ Functional Food Centre, School of Life Sciences, Oxford Brookes University, Gipsy Lane, Headington, \\ Oxford OX3 OBP, UK
}

\begin{abstract}
Advances in public health nutrition rely on the accurate quantification of biologically active constituents of commonly consumed food products. Antioxidants are one particular group of food constituents which are regularly quantified due to their proposed health benefits. Epidemiological studies have shown a reduction in the incidence of disease states such as CVD and cancers when greater amounts of naturally occurring antioxidants are consumed ${ }^{(1)}$. Tomatoes and tomato products have received considerable scientific attention, primarily because they are the major dietary source of the potent antioxidant lycopene and a good source of a variety of other antioxidant carotenoids. Carotenoid bioavailability is increased when tomatoes are processed and therefore, tomato juices may be an excellent source of bioavailable antioxidants ${ }^{(2)}$. In addition to quantifying the total antioxidant capacity (TAC) of produce, it is important to know whether these antioxidants are biologically available after digestion. Therefore, the objective of the present study was to evaluate the TAC of 10 commercially available tomato juices, both value and premium brands, before and after the gastric and duodenal phases of an in vitro digestion. Samples of each juice were subjected to an in vitro digestion procedure involving simulated gastric and duodenal phases. Samples were analysed for TAC before and after the gastric phase, and after the duodenal phase of digestion, using both the ferric ion reducing antioxidant power (FRAP) assay ${ }^{(3)}$ and the 2,2-diphenyl-1-picrylhydrazyl (DPPH) radical scavenging assay ${ }^{(4)}$.
\end{abstract}

\begin{tabular}{|c|c|c|c|c|c|c|c|c|c|c|}
\hline \multicolumn{7}{|c|}{$\operatorname{FRAP}(\mu \mathrm{mol} / \mathrm{l})$} & \multicolumn{2}{|c|}{ DPPH \% inhibition } & \multicolumn{2}{|c|}{ DDPH (fold change) } \\
\hline Juice & Before & SE & Gastric & $\mathrm{SE}$ & Duodenal & $\mathrm{SE}$ & Before & $\mathrm{SE}$ & Gas & Duo \\
\hline 1 & 1971 & 21 & $3668^{*}$ & 298 & $3687^{*}$ & 291 & 79.9 & 1.1 & 1.01 & 0.71 \\
\hline 2 & 2497 & 18 & $3890 *$ & 83 & $3743^{*}$ & 106 & 88.5 & 0.2 & 1.24 & 0.86 \\
\hline 3 & 1843 & 15 & $2841^{*}$ & 71 & $2637 *$ & 106 & 67.3 & 0.9 & 1.02 & 0.7 \\
\hline 4 & 2750 & 111 & $3724 *$ & 120 & $3190 *$ & 89 & 81 & 0.6 & 1.13 & 0.82 \\
\hline 5 & 2905 & 223 & $3882 *$ & 105 & $3308 *$ & 224 & 81.8 & 0.2 & 1.02 & 0.67 \\
\hline 6 & 3138 & 96 & $5008^{*}$ & 159 & $3772 *$ & 208 & 85.7 & 0.1 & 1.21 & 0.76 \\
\hline 7 & 2414 & 88 & $3172 *$ & 87 & $2959 *$ & 126 & 82.5 & 0.2 & 1.26 & 0.92 \\
\hline 8 & 2683 & 74 & $3287^{*}$ & 68 & $3077 *$ & 5 & 81.2 & 0.4 & 1.29 & 0.84 \\
\hline 9 & 2497 & 84 & $3547 *$ & 137 & $3289^{*}$ & 66 & 83.9 & 0.4 & 1.24 & 0.94 \\
\hline 10 & 2202 & 39 & $3417 *$ & 87 & $3111^{*}$ & 51 & 80.4 & 0.4 & 1.15 & 0.88 \\
\hline
\end{tabular}

$n 3$ independent experiments; $* P<0.01=$ significantly different from same sample prior to digestion, $\mathrm{SE}=$ standard error, fold change is shown from sample prior to digestion, for the gastric phase (Gas) and duodenal phase (Duo).

The TAC of the analysed tomato juices increased significantly $(P<0.01)$ after both gastric and duodenal phases as measured by the FRAP assay. However, the results of the DPPH assay showed a small increase in TAC after the gastric phase and a small decrease after the duodenal phase. In conclusion, tomato juices show an equivalent or increased TAC following digestion. This study provides the first measurement of tomato juice antioxidants after digestion.

1. Agarwal A \& Rao AV (2000) Tomato lycopene and its role in human health and chronic diseases. Can Med Assoc J 163, 739-744.

2. Ryan L, O'Connell O, O'Sullivan L et al. (2008) Micellarisation of carotenoids from raw and cooked vegetables. Plant Food Hum Nutr 63, 127-133.

3. Benzie IFF \& Strain JJ (1996) The ferric reducing ability of plasma (FRAP) as a measure of “Antioxidant Power': the FRAP assay. Anal Biochem 239, $70-76$.

4. Brand-Williams W, Cuvelier ME \& Berset C (1995) Kinetics and mechanisms of antioxidant activity using the DPPH• free radical method. LebensmWiss u-Technol 30, 609-615. 